Functiones et Approximatio

52.2 (2015), 229-252

doi: $10.7169 / \mathrm{facm} / 2015.52 .2 .4$

\title{
THE FOURIER EXPANSION OF HECKE OPERATORS FOR VECTOR-VALUED MODULAR FORMS
}

\author{
OLIVER Stein
}

\begin{abstract}
We compute the Fourier expansion of Hecke operators on vector-valued modular forms for the Weil representation associated to a lattice $L$. The Hecke operators considered in this paper include operators $T\left(p^{2 l}\right)$ where $p$ is a prime dividing the level of the lattice $L$. Additionally, an explicit formula for a general type of Gauss sum associated to a lattice $L$ drops out as a by-product.
\end{abstract}

Keywords: Fourier expansion, Hecke operators, vector-valued modular forms, Weil representation, Gauss sums.

\section{Introduction}

Hecke operators are an important part of the theory of modular forms, see e.g. [Sh1], [Sh2], [Sh3] or [EZ] and many other papers. They can be used to study arithmetic properties of Fourier coefficients of a modular form and to exhibit relations between them. To this end an explicit description of the action of Hecke operators on the Fourier coefficients of a modular form is vital.

Borcherds discovered in [Bo1] a theta lift which maps vector-valued modular forms for $\mathrm{SL}_{2}(\mathbb{Z})$ to autormorphic forms on orthogonal groups. These vector-valued modular forms transform with a certain representation of $\mathrm{SL}_{2}(\mathbb{Z})$, the Weil representation $\varrho_{L}$, which is associated to a lattice $L$. Since then vector-valued modular forms of this type are the subject of many recently published papers, see e.g. [Bo2], [Br],[BS], [Sc], [BO1] and [BO2].

The paper [BS] provides a foundation of a Hecke theory for vector-valued modular forms of type $\varrho_{L}$ along the same lines as it is known for the classical elliptic modular forms. Two cases are considered in the paper. The first one defines Hecke operators $T(n)$ where $n$ is assumed to be coprime to the level $N$ of the lattice $L$. The definition of such a Hecke operator involves the extension of the Weil representation to some subgroup of $G L_{2}^{+}(\mathbb{Q})$. This extension depends fundamentally

2010 Mathematics Subject Classification: primary: 11F25, 11F27; secondary: 11LO5, $11 \mathrm{E} 08$ 
on the condition $(n, N)=1$ and assumes further that $n$ is a square modulo $N$. Moreover, for modular forms of half-integral weight it turns out that $T(n)$ is only non trivial if $n$ is a square number in the integers. One important result is the explicit description of the action of $T(n)$ on the Fourier expansion of a modular form for a prime or a square of a prime.

The second case is a generalisation of the first one since the assumption $(n, N)=1$ is dropped. The results of the first case cannot be carried over to the general one since the extension of $\varrho_{L}$ relies essentially on the above mentioned condition on $n$. Nevertheless, it is still possible to extend the Weil representation to a suitable double coset and to define a Hecke operator by the action of such a double coset. In contrast to the first case, a description of the action of $T(n)$ on the Fourier coefficients of a modular form is not given.

The present paper provides an explicit formula for the Fourier expansion of the Hecke operator $T\left(p^{2 l}\right)$ for an odd prime $p$ and a positive integer $l$. Compared to the paper [BS], there are no restrictions on the prime $p$ except that it has to be odd. Insofar, the paper at hand provides the missing action on the Fourier expansion for the more general Hecke operator and a generalisation of the formula for the action of $T\left(p^{2}\right)$ in the first mentioned case above.

We now describe the content of the paper in some more detail. Let $(L, b(\cdot, \cdot))$ be an even non-degenerate lattice of type $\left(b^{+}, b^{-}\right)$where $b(\cdot, \cdot)$ denotes a bilinear form on $L$ with associated quadratic form $x \mapsto q(x)$. The number $b^{+}-b^{-}$is called the signature of $L$. Let $L^{\prime}$ be the dual lattice of $L$ and $\mathcal{L}:=L^{\prime} / L$ the discriminant group. The level of $L$ is defined to be the smallest integer $N$ such that $N q(x) \in \mathbb{Z}$ for all $x \in L^{\prime}$. In order to keep the exposition in the introduction as simple as possible we limit ourselves to the situation where the signature of $L$ is even. In the body of the paper the case of odd and even signature is treated. For even signature the Weil representation is a representation $\varrho_{L}$ of $\Gamma(1)=\mathrm{SL}_{2}(\mathbb{Z})$ on the group ring $\mathbb{C}[\mathcal{L}]$ of the discriminant group,

$$
\varrho_{L}: \Gamma(1) \longrightarrow \mathbb{C}[\mathcal{L}] .
$$

A modular form of weight $k \in \mathbb{Z}$ and type $\varrho_{L}$ for $\Gamma(1)$ is a holomorphic function $f: \mathbb{H} \longrightarrow \mathbb{C}[\mathcal{L}]$ which satisfies

$$
f(\gamma \tau)=(c \tau+d)^{k} \varrho_{L}(\gamma) f(\tau)
$$

for all $\gamma=\left(\begin{array}{ll}a & b \\ c & d\end{array}\right) \in \Gamma(1)$ and which is holomorphic at the cusp $\infty$. A cusp form $f$ satisfies the usual additional condition with respect to the Fourier expansion of all component functions $f_{\lambda}$ of $f=\sum_{\lambda \in \mathcal{L}} f_{\lambda} \mathfrak{e}_{\lambda}$. Here, $\left\{\mathfrak{e}_{\lambda}\right\}_{\lambda \in \mathcal{L}}$ denotes the standard basis of $\mathbb{C}[\mathcal{L}]$ considered as a vector space.

As already stated, this paper is mainly concerned with the calculation of the Fourier expansion of $\left.f\right|_{k, L} T\left(p^{2 l}\right)$ for any odd prime $p$ and any positive integer $l$. We now briefly sketch how $T\left(p^{2 l}\right)$ can be defined (for details see [BS]) and outline the steps necessary to obtain explicit formulas for the action of $T\left(p^{2 l}\right)$ on Fourier expansions. Let $\alpha=\left(\begin{array}{cc}p^{2 l} & 0 \\ 0 & 1\end{array}\right) \in \mathrm{GL}_{2}^{+}(\mathbb{Q})$. We define $\varrho_{L}$ on $\alpha$ by

$$
\varrho_{L}^{-1}(\alpha) \mathfrak{e}_{\lambda}=\mathfrak{e}_{p^{l} \lambda}
$$


It can be proven that (1.1) can be extended to an action on the double coset $\Gamma(1) \alpha \Gamma(1)$ by

$$
\varrho_{L}^{-1}(\beta) \mathfrak{e}_{\lambda}=\varrho_{L}^{-1}\left(\gamma^{\prime}\right) \varrho_{L}^{-1}(\alpha) \varrho_{L}^{-1}(\gamma) \mathfrak{e}_{\lambda}
$$

where $\beta=\gamma \alpha \gamma^{\prime}$ and $\gamma, \gamma^{\prime} \in \Gamma(1)$. The Hecke operator $T\left(p^{2 l}\right)$ is then defined in the usual way by the action of the double coset $\Gamma(1) \alpha \Gamma(1)$.

$$
\left.f\right|_{k, L} T\left(p^{2 l}\right)=p^{2 l(k-2)} \sum_{i} \sum_{\lambda \in \mathcal{L}}\left(\left.f_{\lambda}\right|_{k} \delta_{i}\right) \varrho_{L}^{-1}\left(\delta_{i}\right) \mathfrak{e}_{\lambda}
$$

where the $\delta_{i} \in \mathrm{GL}_{2}^{+}(\mathbb{Q})$ form a set of left coset representatives of $\Gamma(1) \backslash \Gamma(1) \alpha \Gamma(1)$.

In order to calculate the Fourier expansion of $\left.f\right|_{k, L} T\left(p^{2 l}\right)$ it is at first necessary to evaluate

$$
\varrho_{L}^{-1}\left(\delta_{i}\right) \mathfrak{e}_{\lambda}
$$

for all representatives $\delta_{i}$ according to the rule of (1.2). Theorem 5.2 provides the corresponding formulas for $\varrho_{L}^{-1}\left(\delta_{i}\right) \mathfrak{e}_{\lambda}$. The proof of the theorem is based on a formula of Shintani, [Shin], Prop. 1.6, and the formulas of the Weil representation for the standard generators $S$ and $T$ of $\Gamma(1)$. These formulas applied, lead to considerably complicated expressions which are simplified to a "ready to compute" formula in the course of the proof.

In Theorem 5.4 the Fourier expansion of $\left.f\right|_{k, L} T\left(p^{2 l}\right)$ will then be computed. As a corollary the corresponding formula for a prime $p$ coprime to $N$ is given. It can be verified that the Fourier expansion for $l=1$, proven in Theorem 4.10 of [BS], can indeed be recovered by the formula in corollary 5.6. In Corollary 5.7 we show that the vector-valued Hecke operator $T\left(p^{2}\right)$ corresponds to the Hecke operator $T_{p}$ for Jacobi forms by comparing Fourier expansions.

In the course of calculating the expansion of $\left.f\right|_{k, L} T\left(p^{2 l}\right)$ some general Gauss sums

$$
G_{h, s}(L)=\sum_{v \in L / p^{l} L} e\left(\frac{h}{p^{l}} q(v)\right)
$$

occur. There is a separate section, section 4, in this paper which is devoted to explicitly evaluate these Gauss sums. This evaluation is based on a diagonalization of the quadratic form $q$ on the $\left(\mathbb{Z} / p^{l} \mathbb{Z}\right)$-module $L / p^{l} L$ which is similar to the diagonalization over the $p$-adic integers in Theorem 2 in chapter 15 of [CS]. Gauss sums of this type are not new and appear frequently in the literature. The papers [Sc], [Sc1] and [Str] provide also explicit formulas for (1.4) based on the Jordan decomposition of a quadratic module. However, these formulas seem not to be immediately applicable to our needs and look not as simple as ours.

\section{The Weil representation and vector-valued modular forms}

In this section we recall some basic facts about the Weil representation and vectorvalued modular forms transforming with the Weil representation. We follow [BS] and $[\mathrm{Br}]$. 
The group $\mathrm{GL}_{2}^{+}(\mathbb{R})=\left\{M \in \mathrm{GL}_{2}(\mathbb{R}) ; \quad \operatorname{det}(M)>0\right\}$ acts on the upper halfplane $\mathbb{H}=\{\tau=x+i y \in \mathbb{C} ; \quad \operatorname{Im}(\tau)>0\}$ and on $\mathbb{H} \cup \mathbb{R} \cup\{\infty\}$, respectively, by linear fractional transformations. For $z \in \mathbb{C}$ let $\sqrt{z}=z^{1 / 2}$ be the principal branch of the square root, i. e. $\arg (\sqrt{z}) \in(-\pi / 2, \pi / 2]$. For an integer $k$ we put $z^{k / 2}=$ $\sqrt{z}^{k}$. For $M=\left(\begin{array}{ll}a & b \\ c & d\end{array}\right) \in \mathrm{GL}_{2}^{+}(\mathbb{R})$ and $\tau \in \mathbb{H}$ we define the automorphy factor

$$
j(M, \tau)=\sqrt{c \tau+d} .
$$

With $\widetilde{G L}_{2}^{+}(\mathbb{R})$ we denote the metaplectic cover of $\mathrm{GL}_{2}^{+}(\mathbb{R})$, i. e. the group of pairs $(M, \phi(\tau))$, where $M \in \mathrm{GL}_{2}^{+}(\mathbb{R})$ and $\phi(\tau)=j(M, \tau)$ or $-j(M, \tau)$. The group multiplication is given by

$$
\left(M_{1}, \phi_{1}(\tau)\right)\left(M_{2}, \phi_{2}(\tau)\right)=\left(M_{1} M_{2}, \phi_{1}\left(M_{2} \tau\right) \phi_{2}(\tau)\right)
$$

Let $\widetilde{\mathrm{GL}}_{2}^{+}(\mathbb{R}) \rightarrow \mathrm{GL}_{2}^{+}(\mathbb{R})$ be the projection on the first component. For any subgroup $\Gamma$ of $\mathrm{GL}_{2}^{+}(\mathbb{R})$ we write $\widetilde{\Gamma}$ for the inverse image under the projection. In particular, we put $\widetilde{\Gamma}(1)=\widetilde{\mathrm{SL}}_{2}(\mathbb{Z})$. The group $\widetilde{\Gamma}(1)$ is generated by $T=\left(\left(\begin{array}{ll}1 & 1 \\ 0 & 1\end{array}\right), 1\right)$ and $S=\left(\left(\begin{array}{cc}0 & -1 \\ 1 & 0\end{array}\right), \sqrt{\tau}\right)$ satisfying the relations $S^{2}=(S T)^{3}=Z$ where $Z=$ $\left(\left(\begin{array}{cc}-1 & 0 \\ 0 & -1\end{array}\right), i\right)$.

Let $(L, b(\cdot, \cdot))$ be a non-degenerate even lattice of type $\left(b^{+}, b^{-}\right)$, where $b(\cdot, \cdot)$ denotes a bilinear form on $L$ with associated quadratic form $x \mapsto q(x)=\frac{1}{2} b(x, x)$. Further, let $\operatorname{sig}(L)=b^{+}-b^{-}$be the signature of $L$. We define the dual lattice $L^{\prime}$ of $L$ to be

$$
L^{\prime}=\{x \in L \otimes \mathbb{Q}: b(x, y) \in \mathbb{Z} \text { for all } y \in L\} .
$$

Since $L$ is even we have that $L$ is a subgroup of $L^{\prime}$ and

$$
\mathcal{L}:=L^{\prime} / L
$$

is a finite abelian group. The $\bmod 1$ reduction of $b(\cdot, \cdot)$ is a $\mathbb{Q} / \mathbb{Z}$-valued bilinear form on $\mathcal{L}$. The associated quadratic form is the mod 1 reduction of the quadratic form $x \mapsto q(x)$ on $L^{\prime}$. For $n \in \mathbb{N}$ we define the subgroups

$$
\begin{aligned}
& \mathcal{L}^{n}=\{\mu \in \mathcal{L}: \exists \nu \in \mathcal{L} \mu=n \nu\}, \\
& \mathcal{L}_{n}=\{\mu \in \mathcal{L}: n \mu=0\}
\end{aligned}
$$

and the subset

$$
\mathcal{L}^{n *}=\left\{\mu \in \mathcal{L}:(\mu, \nu) \equiv n \nu^{2} / 2(\bmod 1) \text { for all } \nu \in\left(L^{\prime} / L\right)_{n}\right\}
$$

of $\mathcal{L}$. These subgroups are connected by the following exact sequence

$$
0 \longrightarrow \mathcal{L}_{n} \longrightarrow \mathcal{L} \longrightarrow \mathcal{L}^{n} \longrightarrow 0
$$

where the second map is an embedding and the third one the multiplication with $n$. Furthermore, we denote with $L(n)$ and $L^{\prime}(n)$ the lattice $L$ and $L^{\prime}$ respectively 
equipped with the scaled bilinear form $n b(\cdot, \cdot)$ and accordingly the scaled quadratic form $n q(\cdot)$. For the rest of the paper we will use the following notation

$$
\mathcal{L}(n):=L^{\prime}(n) / L(n) .
$$

Note that one can prove that $\mathcal{L}^{n *}=\mathcal{L}^{n}$, if $n$ is an odd integer (see e.g. [Bo2], p. 324).

There is a unitary representation $\varrho_{L}$ of $\widetilde{\Gamma}(1)$ on the group algebra $\mathbb{C}[\mathcal{L}]$ which will be described now: Let $\left\{\mathfrak{e}_{\lambda}\right\}_{\lambda \in \mathcal{L}}$ be the standard basis of $\mathbb{C}[\mathcal{L}]$ as a $\mathbb{C}$-vector space and $e(x)=e^{2 \pi i x}$. It is sufficient to define the representation on the generators $S$ and $T$ :

$$
\begin{aligned}
\varrho_{L}(T)\left(\mathfrak{e}_{\lambda}\right) & =e(q(\lambda)) \mathfrak{e}_{\lambda}, \\
\varrho_{L}(S)\left(\mathfrak{e}_{\lambda}\right) & =\frac{e(-\operatorname{sig}(L) / 8)}{\sqrt{|\mathcal{L}|}} \sum_{\mu \in \mathcal{L}} e(-b(\lambda, \mu)) \mathfrak{e}_{\mu} .
\end{aligned}
$$

The representation on $Z$ is given by

$$
\varrho_{L}(Z)\left(\mathfrak{e}_{\lambda}\right)=e(-\operatorname{sig}(L) / 4) \mathfrak{e}_{-\lambda} .
$$

It can be verified that the formulas (2.3) and (2.2) satisfy the above mentioned relations of $S$ and $T$ which proves that they indeed define a representation on $\widetilde{\Gamma}(1)$, the Weil representation.

We have $Z^{2}=\left(\left(\begin{array}{ll}1 & 0 \\ 0 & 1\end{array}\right),-1\right)$ and $\varrho_{L}\left(Z^{2}\right)\left(\mathfrak{e}_{\lambda}\right)=(-1)^{\operatorname{sig}(L)} \mathfrak{e}_{\lambda}$. Therefore, if the signature of $L$ is even, the Weil representation factors through $\Gamma(1)$. Let $N$ be the level of the lattice $L$, i. e. the smallest positive integer $N$ such that $N q(x) \in \mathbb{Z}$ for all $x \in L^{\prime}$. It is well known that the Weil representation is trivial on the principal congruence subgroup $\Gamma(N)$ (see [Eb], Theorem 3.2). It follows that $\varrho_{L}$ factors through the finite group

$$
\Gamma(1) / \Gamma(N) \cong \mathrm{SL}_{2}(\mathbb{Z} / N \mathbb{Z}) .
$$

If the signature $\operatorname{sig}(L)$ is odd, one can show that 4 divides the level $N$ of the lattice. In this case the Weil representation is trivial on the group $\Gamma(N)^{*}=\{(\gamma, J(\gamma, \tau))$ : $\gamma \in \Gamma(N)\} \subset \widetilde{\Gamma}(1)$ where $J\left(\left(\begin{array}{ll}a & b \\ c & d\end{array}\right), \tau\right)=\left(\frac{c}{d}\right) j\left(\left(\begin{array}{ll}a & b \\ c & d\end{array}\right), \tau\right)$ is the theta multiplier. It follows that $\varrho_{L}$ factors through the finite quotient

$$
\widetilde{\Gamma}(1) / \Gamma(N)^{*},
$$

see [BS], p. 253.

The following proposition provides an explicit formula for the Weil representation on lower triangular matrices of $\widetilde{\Gamma}(1)$. A proof can be found in [BS], Lemma 2.3.

Proposition 2.1. Let $U=\left(\left(\begin{array}{ll}1 & 0 \\ 1 & 1\end{array}\right), \sqrt{\tau+1}\right) \in \widetilde{\Gamma}(1)$. The Weil representation of $U^{m}$ is given by

$$
\varrho_{L}\left(U^{m}\right) \mathfrak{e}_{\lambda}=\frac{1}{|\mathcal{L}|} \sum_{\mu, \nu \in \mathcal{L}} e(-m q(\mu)+b(\mu, \lambda-\nu)) \mathfrak{e}_{\nu}
$$


We now define vector-valued modular forms of type $\varrho_{L}$. With respect to the standard basis of $\mathbb{C}[\mathcal{L}]$ a function $f: \mathbb{H} \rightarrow \mathbb{C}[\mathcal{L}]$ can be written in the form

$$
f(\tau)=\sum_{\lambda \in \mathcal{L}} f_{\lambda}(\tau) \mathfrak{e}_{\lambda}
$$

The following operator generalises the usual Petersson slash operator to the space of all those functions. For $k \in \frac{1}{2} \mathbb{Z}$ we define

$$
\left.f\right|_{k, L}(\gamma, \phi)=\phi(\gamma, \tau)^{-2 k} \varrho_{L}(\gamma, \phi)^{-1} f(\gamma \tau) .
$$

A holomorphic function $f: \mathbb{H} \rightarrow \mathbb{C}[\mathcal{L}]$ is called a modular form of weight $k$ and type $\varrho_{L}$ for $\widetilde{\Gamma}(1)$ if $\left.f\right|_{k, L}(\gamma, \phi)=f$ for all $(\gamma, \phi) \in \widetilde{\Gamma}(1)$, and if $f$ is holomorphic at the cusp $\infty$. Here the last condition means that all Fourier coefficients $c(\lambda, n)$ with $n<0$ vanish. If in addition $c(\lambda, n)=0$ for all $n=0$, we call the corresponding modular form a cusp form. We denote by $M_{k, L}$ the space of all such modular forms, by $S_{k, L}$ the subspace of cusp forms. For more details see e.g. [Br] or [BS]. Note that formula (2.4) implies that $M_{k, L}=\{0\}$ unless

$$
2 k \equiv \operatorname{sig}(L) \quad(\bmod 2) .
$$

Therefore, if the signature of $L$ is even, only non-trivial spaces of integral weight can occur, if the signature of $L$ is odd only non-trivial spaces of half-integral weight can occur. The Petersson scalar product on $S_{k, L}$ is given by

$$
(f, g)=\int_{\Gamma(1) \backslash \mathbb{H}}\langle f(\tau), g(\tau)\rangle \operatorname{Im} \tau^{k} d \mu(\tau)
$$

where

$$
d \mu(\tau)=\frac{d x d y}{y^{2}}
$$

denotes the hyperbolic volume element and

$$
\left\langle\sum_{\lambda \in \mathcal{L}} a_{\lambda} \mathfrak{e}_{\lambda}, \sum_{\lambda \in \mathcal{L}} b_{\lambda} \mathfrak{e}_{\lambda}\right\rangle=\sum_{\lambda \in \mathcal{L}} a_{\lambda} \overline{b_{\lambda}}
$$

is the standard scalar product on the group ring $\mathbb{C}[\mathcal{L}]$.

\section{Hecke operators on vector-valued modular forms}

In this section we briefly recall how Hecke operators on vector-valued modular forms can be defined. All details can be found in [BS]. An alternative approach leading to the same Hecke operator is described in [St]. As usual, Hecke operators will be defined by the action of a suitable Hecke algebra. 


\subsection{The case of even signature}

In order to define Hecke operators on $M_{k, L}$ one has to extend the Petersson slash operator in (2.7) to some suitable group which is isomorphic to a subgroup of $\mathrm{GL}_{2}^{+}(\mathbb{Q})$. In particular, this means that the Weil representation has to be extended to this group. As a starting point the Weil representation, viewed as a representation of the finite group $S(N):=\Gamma(1) / \Gamma(N)$,

$$
\varrho_{L}(A) \mathfrak{e}_{\lambda}=\varrho_{L}(s(A)) \mathfrak{e}_{\lambda}, \quad A \in S(N),
$$

can be extended to a group isomorphic to a subgroup of $\mathrm{GL}_{2}(\mathbb{Z} / N \mathbb{Z})$. Here $s: S(N) \rightarrow \Gamma(1)$ is a section, that is $\pi_{N} \circ s=\mathrm{id}_{S(N)}$, where $\pi_{N}$ denotes the component-wise reduction modulo $N$. Let $Q(N)$ be the group

$$
Q(N)=\left\{(M, r) \in \mathrm{GL}_{2}(\mathbb{Z} / N \mathbb{Z}) \times(\mathbb{Z} / N \mathbb{Z})^{*}: \operatorname{det}(M) \equiv r^{2}(\bmod N)\right\}
$$

with the product defined component-wise. By $M \mapsto(M, 1)$ the group $S(N)$ can be embedded into $Q(N)$. Moreover, for $(M, r) \in Q(N)$ the map $(M, r) \mapsto$ $\left(M\left(\begin{array}{ll}r & 0 \\ 0 & r\end{array}\right)^{-1}, r\right)$ defines an isomorphism $Q(N) \cong S(N) \times(\mathbb{Z} / N \mathbb{Z})^{*}$. Then the Weil representation of $(\mathbb{Z} / N \mathbb{Z})^{*}$ can be defined as follows

$$
\varrho_{L}\left(\left(\begin{array}{ll}
r & 0 \\
0 & r
\end{array}\right), r\right) \mathfrak{e}_{\lambda}=\frac{g_{1}(L)}{g_{r}(L)} \mathfrak{e}_{\lambda}
$$

and on the whole group $Q(N)$ by

$$
\varrho_{L}(M, r) \mathfrak{e}_{\lambda}=\varrho_{L}\left(M\left(\begin{array}{cc}
r & 0 \\
0 & r
\end{array}\right)^{-1}, 1\right) \circ \varrho_{L}\left(\left(\begin{array}{cc}
r & 0 \\
0 & r
\end{array}\right), r\right) \mathfrak{e}_{\lambda} .
$$

Here, $g_{r}(L)$ is a Gauss sum defined by

$$
g_{r}(L)=\sum_{\lambda \in \mathcal{L}} e(r q(\lambda))
$$

Since the assignment $r \mapsto \frac{g_{1}(L)}{g_{r}(L)}$ defines a character of $(\mathbb{Z} / N \mathbb{Z})^{*}$ (see [BS], p. 256) we have that (3.2) is indeed a representation. It is easily seen that (3.3) extends the Weil representation to a representation of $Q(N)$. Consider the groups

$$
\begin{aligned}
\mathcal{G}(N)= & \left\{M \in \mathrm{GL}_{2}^{+}(\mathbb{Q}): \exists n \in \mathbb{Z} \text { with }(n, N)=1 \text { such that } n M \in M_{2}(\mathbb{Z})\right. \\
& \text { and }(\operatorname{det}(n M), N)=1\}
\end{aligned}
$$

and

$$
\mathcal{Q}(N)=\left\{(M, r) \in \mathcal{G}(N) \times(\mathbb{Z} / N \mathbb{Z})^{*}: \operatorname{det}(M) \equiv r^{2}(\bmod N)\right\} .
$$

The modular group $\Gamma(1)$ can be embedded into $\mathcal{Q}(N)$ by $\gamma \mapsto(\gamma, 1)$. The component-wise reduction $\pi_{N}$ maps the group $\mathcal{Q}(N)$ onto the group $Q(N)$. Therefore, the Weil representation can be extended to the group $\mathcal{Q}(N)$ by

$$
\varrho_{L}: \mathcal{Q}(N) \longrightarrow \mathrm{GL}(\mathbb{C}[\mathcal{L}]), \quad(M, r) \mapsto \varrho_{L}\left(\pi_{N}(M), r\right),
$$


where $\varrho_{L}$ on $Q(N)$ is defined by (3.3). The action of $\mathcal{Q}(N)$ on vector-valued functions is then given by

$$
\left.f\right|_{k, L}(M, r)=\sum_{\lambda \in \mathcal{L}}\left(\left.f_{\lambda}\right|_{k} M\right) \varrho_{L}^{-1}(M, r) \mathfrak{e}_{\lambda}
$$

where $f=\sum_{\lambda \in \mathcal{L}} f_{\lambda} \mathfrak{e}_{\lambda}$ and

$$
\left.f\right|_{k} M=\phi(M, \tau)^{-2 k} f(M \tau)
$$

is the usual Petersson slash operator. It is easily seen that (3.7) extends the action (2.7) of $\Gamma(1)$ to the group $\mathcal{Q}(N)$.

\subsection{The case of odd signature}

If the signature of $L$ is odd, it suffices to consider vector-valued modular forms of half-integral weight. In this case (3.8) and (3.6) define only projective actions of the group $\mathcal{Q}(N)$. In order to obtain honest actions one needs to introduce appropriate central extensions of $\mathcal{Q}(N)$. First we consider the action on $\mathbb{C}[\mathcal{L}]$. As already mentioned (3.6) yields only a projective representation, that is

$$
\varrho_{L}: \mathcal{Q}(N) \longrightarrow \mathrm{GL}(\mathbb{C}[\mathcal{L}]) /\{ \pm 1\}, \quad g \mapsto \varrho_{L}(g) .
$$

Choosing a section $s: \mathrm{GL}(\mathbb{C}[\mathcal{L}]) /\{ \pm 1\} \rightarrow \mathrm{GL}(\mathbb{C}[\mathcal{L}])$ gives rise to a cocycle $c:$ $\mathcal{Q}(N) \times \mathcal{Q}(N) \rightarrow\{ \pm 1\}$ and a central group extension

$$
\mathcal{Q}_{1}(N)=\mathcal{Q}(N) \times\{ \pm 1\}
$$

By setting

$$
\varrho_{L}(M, r, t)=t \varrho_{L}(M, r)
$$

for $(M, r, t) \in \mathcal{Q}_{1}(N)$ we obtain a representation of $\mathcal{Q}_{1}(N)$. For $(\gamma, 1) \in \Gamma(1) \times$ $\{1\} \subset \mathcal{Q}(N)$ we set

$$
\varrho_{L}(\gamma, 1)=\varrho_{L}(\gamma, j(\gamma, \tau))
$$

This choice of $s$ yields an injective homomorphism of $\widetilde{\Gamma}(1)$ into $\mathcal{Q}_{1}(N)$

$$
\widetilde{\Gamma}(1) \longrightarrow \mathcal{Q}_{1}(N), \quad(\gamma, \pm j(\gamma, \tau)) \mapsto(\gamma, 1, \pm 1) .
$$

Moreover, we set

$$
\varrho_{L}\left(\left(\begin{array}{cc}
m^{2} & 0 \\
0 & 1
\end{array}\right), m\right) \mathfrak{e}_{\lambda}=\mathfrak{e}_{m^{-1} \lambda} .
$$

On the other hand, if the weight $k \in \mathbb{Z}+\frac{1}{2}$ the action (3.8) defines a cocyle which is determined by the square root of the automorphic factor. One can show that this cocyle is not cohomologous to the cocycle $c$ on the group $\mathcal{Q}(N)$. However, the choice (3.9) shows that they are identical on the group $\widetilde{\Gamma}(1)$ and by a different choice of $s$ cohomologous. 
Therefore, in order to define an action on vector-valued functions one has to define a twofold central group extension

$$
\begin{aligned}
& \mathcal{Q}_{2}(N)=\left\{(M, \phi(M, \tau), r, t): M \in \mathcal{G}(N), r \in(\mathbb{Z} / N \mathbb{Z})^{*},\right. \\
&\left.\operatorname{det}(M) \equiv r^{2} \bmod N, t \in\{ \pm 1\}\right\}
\end{aligned}
$$

of $\mathcal{Q}(N)$ by $\{ \pm 1\}$. Because of (3.10) there is an injective homomorphism

$$
L: \widetilde{\Gamma}(1) \longrightarrow \mathcal{Q}_{2}(N), \quad(\gamma, \pm j(\gamma, \tau)) \mapsto(\gamma, \pm j(\gamma, \tau), 1, \pm 1) .
$$

For an element $(M, \phi(M, \tau), r, t) \in \mathcal{Q}_{2}(N)$ we set

$$
\varrho_{L}(M, \phi(M, \tau), r, t)=\varrho_{L}(M, r, t),
$$

that is, we compose the projection to the group $\mathcal{Q}_{1}(N)$ with the Weil representation on that group. By the definition of the embedding $L$ we have

$$
\varrho_{L}(L(\gamma))=\varrho_{L}(\gamma)
$$

for $\gamma \in \widetilde{\Gamma}(1)$. Note that the Weil representation of $\mathcal{Q}(N)$ and $\mathcal{Q}_{2}(N)$ is unitary with respect to the scalar product (2.10). The action of $\mathcal{Q}_{2}(N)$ on vector-valued functions $f=\sum_{\lambda \in \mathcal{L}} f_{\lambda} \mathfrak{e}_{\lambda}$ is given by

$$
\left.f\right|_{k, L}(M, \phi(M, \tau), r, t)=\sum_{\lambda \in \mathcal{L}}\left(\left.f_{\lambda}\right|_{k}(M, \phi)\right) \varrho_{L}^{-1}(M, r, t) \mathfrak{e}_{\lambda}
$$

where $\left.f\right|_{k}(M, \phi)$ is defined as in (3.8).

\subsection{Hecke Operators}

Let $n \in \mathbb{N}$ be coprime to the level $N$ of $L$. The Hecke operator $T(n)$ will be defined in terms of the action of the Hecke algebra given by the pair of groups $(\mathcal{Q}(N), \Gamma(1) \times\{1\})$ or $\left(\mathcal{Q}_{2}(N), L(\widetilde{\Gamma}(1))\right)$ depending on the parity of the signature of the lattice $L$. The definition of the group $\mathcal{Q}(N)$ implies that we have to assume that $n$ is a square modulo $N$. Further, if the signature of $L$ is odd one can show that $T(n)$ is zero unless $n$ is a square in $\mathbb{Z}$, see [BS], Prop. 4.9. Therefore, we assume that $(n, N)=1$ and

$$
\begin{array}{ll}
n \equiv r^{2} \bmod N, & \text { if } \operatorname{sig}(L) \text { is even, } \\
n=m^{2}, & \text { if } \operatorname{sig}(L) \text { is odd. }
\end{array}
$$

In order to keep the notation as simple as possible and to treat the cases of odd and even signature in the following definition simultaneously we introduce the following notation:

$$
\Gamma= \begin{cases}\Gamma(1) \times\{1\} \subset \Gamma(1) \times(\mathbb{Z} / N \mathbb{Z})^{*}, & \text { if } \operatorname{sig}(L) \text { even }, \\ L(\widetilde{\Gamma}(1)), & \text { if } \operatorname{sig}(L) \text { odd },\end{cases}
$$


where $L$ denotes the embedding (3.13) and accordingly

$$
\mathcal{M}(n)= \begin{cases}\Gamma\left(\left(\begin{array}{ll}
n & 0 \\
0 & 1
\end{array}\right), r\right) \Gamma, & \text { if } \operatorname{sig}(L) \text { even } \\
\Gamma\left(\left(\begin{array}{ll}
n & 0 \\
0 & 1
\end{array}\right), 1, m, 1\right) \Gamma, & \text { if } \operatorname{sig}(L) \text { odd }\end{cases}
$$

As usual, the Hecke operator is then defined by

$$
\left.f\right|_{k, L} T(n)=\left.n^{k / 2-1} \sum_{\widetilde{M} \in \Gamma \backslash \mathcal{M}(n)} f\right|_{k, L} \widetilde{M},
$$

where the slash operator is given by (3.7) and (3.15) depending on the parity of the signature of $L$.

\subsection{Generalized Hecke operators}

In this section we recall from [BS], section 5, the definition of Hecke operators $T(n)$ for all $n \in \mathbb{N}$, in particular for $(n, N)>1$. In the latter case we need to define an extension for the Weil representation to matrices $M \in M_{2}(\mathbb{Z})$ with $(\operatorname{det}(M), N)>$ 1 , since the reduction of $M$ modulo $N$ does not belong to $\mathrm{GL}_{2}(\mathbb{Z} / N \mathbb{Z})$. We use for the rest of this section the following notation

$$
\left.\mathfrak{e}_{\lambda}\right|_{L} M=\varrho_{L}^{-1}(M) \mathfrak{e}_{\lambda}
$$

to define a right action of the double coset $\tilde{\Gamma}(1)\left(\left(\begin{array}{cc}n^{2} & 0 \\ 0 & 1\end{array}\right)\right) \tilde{\Gamma}(1)$. In consistency with the definition of the Weil representation for $(n, N)=1$ we set

$$
\left.\mathfrak{e}_{\lambda}\right|_{L}\left(\left(\begin{array}{cc}
n^{2} & 0 \\
0 & 1
\end{array}\right), 1\right)=\mathfrak{e}_{n \lambda},
$$

and use in the following the abbreviation

$$
\alpha=\left(\left(\begin{array}{cc}
n^{2} & 0 \\
0 & 1
\end{array}\right), 1\right) \in{\widetilde{\mathrm{GL}_{2}^{+}}}^{+}(\mathbb{Q})
$$

(see [BS], (5.1)). The action $(3.21)$ can be extended to the double coset $\tilde{\Gamma}(1) \alpha \tilde{\Gamma}(1)$ in the following way

$$
\left.\mathfrak{e}_{\lambda}\right|_{L} \delta=\left.\left.\left.\mathfrak{e}_{\lambda}\right|_{L} \gamma\right|_{L} \alpha\right|_{L} \gamma^{\prime}
$$

for $\delta=\gamma \alpha \gamma^{\prime} \in \tilde{\Gamma}(1) \alpha \tilde{\Gamma}(1)$. One can show that (3.22) is independent of the decomposition of $\delta$, see [BS], Proposition 5.1, and moreover, indeed defines an action on $\tilde{\Gamma}(1) \alpha \tilde{\Gamma}(1)$, see [BS], Proposition 5.4.

Using (3.21) and (3.22) it is possible to define Hecke operators for all $n \in \mathbb{N}$.

Definition 3.1. Let $n$ be a positive integer and $\alpha=\left(\left(\begin{array}{cc}n^{2} & 0 \\ 0 & 1\end{array}\right), 1\right) \in \widetilde{G L}_{2}^{+}(\mathbb{Q})$. Let

$$
\widetilde{\Gamma}(1) \cdot \alpha \cdot \widetilde{\Gamma}(1)=\bigcup_{i} \widetilde{\Gamma}(1) \cdot \delta_{i}
$$


be a disjoint left coset decomposition. We define the Hecke operator $T\left(n^{2}\right)$ on modular forms $f \in M_{k, L}$ by

$$
\left.f \mapsto f\right|_{k, L} T\left(n^{2}\right)=\left.n^{k-2} \sum_{i} f\right|_{k, L} \delta_{i}=n^{k-2} \sum_{i} \sum_{\lambda \in \mathcal{L}}\left(\left.f_{\lambda}\right|_{k} \delta_{i}\right)\left(\left.\mathfrak{e}_{\lambda}\right|_{L} \delta_{i}\right) .
$$

Note that definition 3.1 generalises the one for the case $(n, N)=1$.

The following theorem is proved in [BS], Theorem 5.6.

Theorem 3.2. Let $m, n \in \mathbb{N}$ be coprime. Then the Hecke operator $T\left(n^{2}\right)$ is a linear operator on $M_{k, L}$ taking cusp forms to cusp forms. It is self-adjoint with respect to the Petersson scalar product (2.9). Moreover,

$$
T\left(m^{2}\right) T\left(n^{2}\right)=T\left(m^{2} n^{2}\right) .
$$

\section{Gauss sums}

In this section we present explicit formulas for some Gauss sums which appear during the calculation of the Fourier expansion of the above defined Hecke operators. Some of these formulas may be known to the expert, others appear in a different form in the literature. For reasons of completeness and because these formulas could be interesting in its own right we provide them here. For the rest of the paper we denote for any prime $p$ by $v_{p}$ the $p$-adic valuation on $\mathbb{Q}$.

Lemma 4.1. Let $p$ be an odd prime, $\chi_{p}$ the character defined by the Legendre symbol $(\dot{\bar{p}})$ and $\chi$ the trivial character. Then for $m, s \in \mathbb{Z}$ with $s>0$,

$g\left(p^{s}, \chi_{p}, m\right):=\sum_{h \in \mathbb{Z} / p^{s} \mathbb{Z}} \chi_{p}(h) e\left(\frac{h m}{p^{s}}\right)= \begin{cases}p^{s-1} \sqrt{p} \epsilon_{p}\left(\frac{m / p^{s-1}}{p}\right), & \text { if } v_{p}(m)=s-1, \\ 0, & \text { otherwise, }\end{cases}$

and

$$
g\left(p^{s}, \chi, m\right):=\sum_{h \in \mathbb{Z} / p^{s} \mathbb{Z}} \chi(h) e\left(\frac{h m}{p^{s}}\right)= \begin{cases}p^{s}-p^{s-1}, & \text { if } p^{s} \mid m, \\ -p^{s-1}, & \text { if } v_{p}(m)=s-1, \\ 0 & \text { otherwise. }\end{cases}
$$

Proof. In the first case

$$
\begin{aligned}
\sum_{h \in \mathbb{Z} / p^{s} \mathbb{Z}} \chi_{p}(h) e\left(\frac{h m}{p^{s}}\right) & =\sum_{h^{\prime} \in \mathbb{Z} / p \mathbb{Z}} \chi_{p}\left(h^{\prime}\right) e\left(\frac{h^{\prime} m}{p^{s}}\right) \sum_{h^{\prime \prime} \in \mathbb{Z} / p^{s-1} \mathbb{Z}} e\left(\frac{h^{\prime \prime} m}{p^{s-1}}\right) \\
& = \begin{cases}p^{s-1} \sum_{h^{\prime} \in \mathbb{Z} / p \mathbb{Z}} \chi\left(h^{\prime}\right)_{p} e\left(\frac{h^{\prime} m}{p^{s}}\right) & \text { if } p^{s-1} \mid m \\
0 & \text { otherwise . }\end{cases}
\end{aligned}
$$

Here, we have used, that $h=h^{\prime}+p h^{\prime \prime}$ runs exactly once through a complete set of representatives of $\mathbb{Z} / p^{s} \mathbb{Z}$ if $h^{\prime}$ runs exactly once through $\mathbb{Z} / p \mathbb{Z}$ and $h^{\prime \prime}$ through $\mathbb{Z} / p^{s-1} \mathbb{Z}$.

The second identity can be found in [BE], (1.6.4). 
Lemma 4.2. Let $p$ be an odd prime, $h$ coprime to $p$ and $r, s \in \mathbb{Z}_{>0}$ with $r \geqslant s$. Then

$$
\sum_{x \in \mathbb{Z} / p^{r} \mathbb{Z}} e\left(\frac{h}{p^{s}} x^{2}\right)=p^{r-s}\left(\frac{h}{p^{s}}\right) \varepsilon_{p^{s}} \sqrt{p^{s}}
$$

where

$$
\varepsilon_{m}=\left\{\begin{array}{lll}
1 & m \equiv 1 & (\bmod 4), \\
i & m \equiv 3 & (\bmod 4)
\end{array}\right.
$$

for $m \in \mathbb{Z}_{>0}$.

Proof. It is easy to verify that if $x$ runs once through a complete set of representatives of $\mathbb{Z} / p^{s} \mathbb{Z}$ and $y$ through a complete set of representatives of $\mathbb{Z} / p^{r-s} \mathbb{Z}$ then $x+p^{s} y$ runs exactly one time through a set of representatives of $\mathbb{Z} / p^{r} \mathbb{Z}$. By means of this representation and the Theorem 1.52 of [BE] the result follows.

For a module $M$ we mean by $M=M_{1} \perp M_{2}$ that $M$ is the direct sum of the submodules $M_{1}$ and $M_{2}$ and that $b\left(M_{1}, M_{2}\right)=0$, where $b$ is a bilinear form on $M$.

Lemma 4.3. Let $L$ be a lattice, $b: L \times L \rightarrow \mathbb{Z}$ a non-degenerate, integral and even bilinear form, let $p$ be an odd prime and $l \in \mathbb{Z}_{>1}$. Then the following statements hold:

(a) The $\mathbb{Z} / p^{l} \mathbb{Z}$-module $L / p^{l} L$ can be decomposed into $\mathbb{Z} / p^{l} \mathbb{Z}$-sub modules

$$
L / p^{l} L=L_{1} \perp L_{2} \perp \cdots \perp L_{r} \perp M
$$

where $L_{i}=\left(\mathbb{Z} / p^{l} \mathbb{Z}\right) u_{i}$ is one-dimensional with $b\left(u_{i}, u_{i}\right) \in\left(\mathbb{Z} / p^{l} \mathbb{Z}\right)^{*}$ for all $i$ and $b(M, M) \subset\left(Z / p^{l} \mathbb{Z}\right) \backslash\left(\mathbb{Z} / p^{l} \mathbb{Z}\right)^{*}$.

(b) The submodule $M$ in the orthogonal decomposition (4.4) allows itself the following orthogonal decomposition in the above defined sense

$$
M=M_{1} \perp M_{2} \perp \cdots \perp M_{s} \perp N
$$

where $M_{j}=\left(\mathbb{Z} / p^{l} \mathbb{Z}\right) v_{j}$ with $b\left(v_{j}, v_{j}\right) \in p^{k}\left(\mathbb{Z} / p^{l} \mathbb{Z}\right)^{*}, k \in\{1, \ldots l-1\}$ and $b(N, N) \equiv 0 \bmod p^{l}$

Proof. (a) Let $B:=\left\{b_{1}, \ldots, b_{D}\right\}$ be a basis of $L$. Then

$$
\left\{x_{1} b_{1}+\cdots x_{D} b_{D}: x_{i} \in \mathbb{Z} / p^{l} \mathbb{Z}\right\}
$$

is a set of representatives of $L / p^{l} L$, and $B$ is also a basis of the $\mathbb{Z} / p^{l} \mathbb{Z}$-module $L / p^{l} L$. Since $\mathbb{Z} / p^{l} \mathbb{Z}$ is a local ring the Theorem 4.1 of $[\mathrm{KS}]$ can be applied which gives immediately the desired result.

(b) The proof is basically the same as in the case of a vector space, see e.g. Theorem 1.20 of $[\mathrm{KS}]$. A similar proof for $p$-adic integers in terms of the Gram matrix can be found in chapter 15, Theorem 2, of [CS]. 
We proceed by induction on the dimension of $M$. If $b(M, M) \equiv 0 \bmod p^{l}$ then nothing is to prove $($ set $N:=M)$. Otherwise, since $2 \in\left(\mathbb{Z} / p^{l} \mathbb{Z}\right)^{*}$, there exists a vector $v \in M$ with $b(v, v) \neq 0$. Since $M$ is finite we can choose $v$ to have minimal $p$-adic valuation $v_{p}(b(v, v))$. We can assume that $v_{p}(b(v, v)) \leqslant v_{p}(b(v, w))$ for all $w \in M$. For, if there exists a $w \in M$ with $0 \neq v_{p}(b(v, w))<v_{p}(b(v, v))$, it can be easily shown that for the element $v+w$ we have $v_{p}(b(v+w, v+w))=v_{p}(b(v, w))$. Now, define $M_{1}:=\left(\mathbb{Z} / p^{l} \mathbb{Z}\right) v$, where $v$ is chosen as above. It remains to prove that $M$ can be written as a direct sum of $M_{1}$ and $M_{1}^{\perp}$. Let $m \in M$. Clearly, $m=a v+(m-a v)$ for any $a \in \mathbb{Z} / p^{l} \mathbb{Z}$. We need to determine an $a \in \mathbb{Z} / p^{l} \mathbb{Z}$ such that $m-a v \in M_{1}^{\perp}$. It suffices to choose $a$ to satisfy the condition

$$
b(v, m-a v)=b(v, m)-a b(v, v)=0 .
$$

This equation can be solved by

$$
a=p^{v_{p}(b(v, m))-v_{p}(b(v, v))} b^{*}(v, m)\left(b^{*}(v, v)\right)^{-1}
$$

where $b^{*}(v, m)$ and $b^{*}(v, v)$ is the part of $b(v, m)$ and $b(v, v)$ respectively which is coprime to $p$. Note that $a$ is a well defined element of $\mathbb{Z} / p^{l} \mathbb{Z}$ by the choice of $v$. If we apply the above explained algorithm to the vectors of the basis $B$ we indeed obtain a direct orthogonal decomposition as claimed $\left(v\right.$ is chosen to be some $b_{j}$ or $b_{j}+b_{i}$ and the orthogonal complement is then build up by the vectors $b_{k}-a_{k} b_{j}$ where $a_{k}$ is formed as in (4.6) and so forth).

Remark 4.4. A well known decomposition of finite $\mathbb{Z}$-modules is the Jordan decomposition. This decomposition assumes that the bilinear form on the module is non-degenerate, see e.g. [Sc1]. Although we assume that $b$ is non-degenerate on the lattice $L$ it is not clear (to me) that same holds for $L / p^{l} L$. However, it was pointed out to me by the referee that $L / p^{l} L$, viewed as a discriminant form, can be described by non-degenerate discriminant forms which in turn can be decomposed into Jordan blocks. Using this fact, our results of this section could also be obtained by the Scheithauers results in [Sc1] and [Sc2].

Lemma 4.5. Let $p$ be an odd prime, $h \in \mathbb{Z}$ coprime to $p$ and $l \in \mathbb{Z}$ with $l>0$. Let $L$ be a non-degenerate even lattice of dimension $D$ and $\left\{v_{1}, \ldots, v_{D}\right\}$ a basis of the $\left(\mathbb{Z} / p^{l} \mathbb{Z}\right)$ - module $L / p^{l} L$ for which $L / p^{l} L$ decomposes into one dimensional sub modules as described in (4.4) and (4.5). Then

(a)

$$
\sum_{v \in L / p^{l} L} e\left(\frac{h}{p^{l}} q(v)\right)=\prod_{k=0}^{l-1}\left(\frac{h}{p^{l-k}}\right)^{n_{k}} \sum_{v \in L / p^{l} L} e\left(\frac{1}{p^{l}} q(v)\right)
$$

and

(b)

$$
\sum_{v \in L / p^{l} L} e\left(\frac{1}{p^{l}} q(v)\right)=p^{l n_{l}} \prod_{k=0}^{l-1}\left(p^{k} \varepsilon_{p^{l-k}} \sqrt{p^{l-k}}\right)^{n_{k}} \prod_{i=1}^{n_{k}}\left(\frac{b^{*}\left(v_{i}, v_{i}\right) / 2}{p^{l-k}}\right)
$$


where $n_{k}$ is the number of integers $b\left(v_{i}, v_{i}\right), i=1, \ldots, d$ with $v_{p}\left(b\left(v_{i}, v_{i}\right)\right)=k$ and $b^{*}\left(v_{i}, v_{i}\right)$ is defined as in the proof of Lemma 4.3. The second product in (4.8) runs over all vectors $v_{i}$ with $v_{p}\left(b\left(v_{i}, v_{i}\right)\right)=k$.

Proof. (a) In view of the orthogonal decompositions (4.4) and (4.5) and Lemma 4.2 we obtain for the Gauss sum

$$
\begin{aligned}
\sum_{v \in L / p^{l} L} e\left(\frac{h}{p^{l}} q(v)\right) & =\sum_{\left(x_{i}\right) \in\left(\mathbb{Z} / p^{l} \mathbb{Z}\right)^{D}} e\left(\frac{h}{p^{l}}\left(\sum_{i=1}^{D} x_{i}^{2}\left(b\left(v_{i}, v_{i}\right) / 2\right)\right)\right) \\
& =\prod_{i=1}^{D}\left(\sum_{x \in \mathbb{Z} / p^{l} \mathbb{Z}} e\left(\frac{h\left(p^{v_{p}\left(b\left(v_{i}, v_{i}\right)\right)} b^{*}\left(v_{i}, v_{i}\right) / 2\right) x^{2}}{p^{l}}\right)\right) \\
& =\prod_{k=0}^{l-1}\left(\frac{h}{p^{l-k}}\right)^{n_{k}} \sum_{v \in L / p^{l} L} e\left(\frac{1}{p^{l}} q(v)\right)
\end{aligned}
$$

where

$$
n_{k}=\left|\left\{b\left(v_{i}, v_{i}\right), i=1, \ldots, D: v_{p}\left(b\left(v_{i}, v_{i}\right)\right)=k\right\}\right| .
$$

(b) Starting with the second expression of (4.9) we obtain

$$
\begin{aligned}
& p^{l n_{l}} \prod_{i=1}^{D-n_{l}}\left(\sum_{x \in \mathbb{Z} / p^{l} \mathbb{Z}} e\left(\frac{\left(b^{*}\left(v_{i}, v_{i}\right) / 2\right) x^{2}}{p^{l-v_{p}\left(b\left(v_{i}, v_{i}\right)\right)}}\right)\right) \\
& =p^{l n_{l}} \prod_{k=0}^{l-1}\left(p^{k} \varepsilon_{p^{l-k}} \sqrt{p^{l-k}}\right)^{n_{k}} \prod_{i=1}^{n_{k}}\left(\frac{b^{*}\left(v_{i}, v_{i}\right) / 2}{p^{l-k}}\right)
\end{aligned}
$$

where we have used Lemma 4.2 to evaluate the quadratic Gauss sums on the left-hand side of (4.10).

Remark 4.6. I have written a PARI/GP-program to evaluate the formula (4.8) including the diagonalization of the Gram-matrix of the lattice $L$ in the sense of Lemma 4.3.

Corollary 4.7. Let $L, p, l, h$ and $D$ as in Lemma 4.5. Further, assume that $p$ is coprime to the level $N$ of the lattice $L$. Then

$$
\begin{aligned}
\sum_{v \in L / p^{l} L} e\left(\frac{h}{p^{l}} q(v)\right) & =\left(\left(\frac{h}{p^{l}}\right) \varepsilon_{p^{l}} \sqrt{p^{l}}\right)^{D} \prod_{i=1}^{D}\left(\frac{b\left(v_{i}, v_{i}\right) / 2}{p^{l}}\right) \\
& =\left(\left(\frac{h}{p^{l}}\right) \varepsilon_{p^{l}} \sqrt{p^{l}}\right)^{D}\left(\frac{(-1)^{b^{-}}|\mathcal{L}|}{p^{l}}\right)\left(\frac{2}{p^{l}}\right)^{D} .
\end{aligned}
$$

Proof. It is well known that each prime dividing $N$ also divides the determinant $\operatorname{det}(L)$ of the Gram-matrix, see [Bu], p. 12. Therefore, all diagonal entries $b\left(v_{i}, v_{i}\right)$ 
of the Gram-matrix with respect to an orthogonal decomposition in the sense of Lemma 4.3 are coprime to $p$, since $\operatorname{det}(L) \equiv \prod_{i=1}^{D} b\left(v_{i}, v_{i}\right)\left(\bmod p^{l}\right)$. It follows that $n_{0}=D$ and $n_{k}=0$ for all $k=1, \ldots, l$. The formulas (4.7) and (4.8) with these values for $n_{k}$ lead to the desired result.

\section{Computation of the Fourier expansion}

\subsection{The Fourier expansion of $T\left(p^{2 l}\right)$}

Let $p$ be an odd prime. We now want to compute the Fourier expansion of $T\left(p^{2 l}\right)$.

Denote with $\alpha \in \widetilde{\mathrm{GL}}_{2}^{+}(\mathbb{Q})$ the matrix $\left(\left(\begin{array}{cc}p^{2 l} & 0 \\ 0 & 1\end{array}\right), 1\right)$, with $\beta_{h, s}$ the matrix $\left(\left(\begin{array}{cc}p^{2 l-s} & h \\ 0 & p^{s}\end{array}\right), \sqrt{p^{s}}\right)$ and with $\gamma_{b}$ the matrix $\left(\left(\begin{array}{cc}1 & b \\ 0 & p^{2 l}\end{array}\right), p^{l}\right)$.

It is well known that a decomposition of $\widetilde{\Gamma}(1) \alpha \widetilde{\Gamma}(1)$ into left cosets is given by

$$
\widetilde{\Gamma}(1) \alpha \widetilde{\Gamma}(1)=\widetilde{\Gamma}(1) \alpha \cup \bigcup_{s=1}^{2 l-1} \bigcup_{h \in\left(\mathbb{Z} / p^{s} \mathbb{Z}\right)^{*}} \widetilde{\Gamma}(1) \beta_{h, s} \cup \bigcup_{b \in \mathbb{Z} / p^{2 l} \mathbb{Z}} \widetilde{\Gamma}(1) \gamma_{b} .
$$

Proposition 5.1. Let $p$ be an odd prime, $l$ a positive integer and $b \in \mathbb{Z} / p^{2 l} \mathbb{Z}$. Then the action of $\gamma_{b}$ in the Weil representation can be computed by

$$
\left.\mathfrak{e}_{\lambda}\right|_{L} \gamma_{b}=\sum_{\substack{\nu \in \mathcal{L} \\ p^{\nu} \nu=\lambda}} e(-b q(\nu)) \mathfrak{e}_{\nu}
$$

Proof. The proof for the case $l=1$ can be found within the proof of Theorem 14.2.9, p. 106, in [St]. The proof for $l>1$ carries over immediately.

Theorem 5.2. Let $p$ be an odd prime, $s, l$ positive integers with $s<2 l$ and $h \in\left(\mathbb{Z} / p^{s} \mathbb{Z}\right)^{*}$. Further, let $D=\operatorname{dim}(L)$ and $G_{h, s}(L)=\sum_{v \in L / p^{s} L} e\left(-\frac{h}{p^{s}} q(v)\right)$. Then

$$
\left.\mathfrak{e}_{\lambda}\right|_{L} \beta_{h, s}=\left\{\begin{array}{cc}
\delta(\lambda, s) p^{-s D / 2} e\left(-\frac{h}{p^{s}} q(\lambda)\right) G_{h, s}(L) \mathfrak{e}_{p^{l-s} \lambda}, & \text { if } l \geqslant s, \\
\delta(\lambda, l)\left|\mathcal{L}_{p^{s-l}}\right|^{-1} p^{-s D / 2} e\left(\frac{h}{p^{s}} q(\lambda)\right) G_{h, s}(L) & \\
\times \sum_{\substack{\rho \in \mathcal{L} \\
p^{s-l} \rho=\lambda}} e\left(b\left(-\rho, \frac{h \lambda}{p^{l}}\right)\right) \mathfrak{e}_{\rho}, & \text { if } l<s,
\end{array}\right.
$$

where $\delta(\lambda, x)$ is 1 if $\lambda \in \mathcal{L}^{p^{x}}$ and 0 otherwise.

Remark 5.3. Let $m$ be $\min (s, l)$. If $\lambda \in \mathcal{L}^{p^{m}}$ then $\frac{1}{p^{s}} q(\lambda)=p^{2 m-s} q\left(\lambda / p^{m}\right)$ and $p^{l-s} b(\lambda, \nu)=p^{l+m-s} b\left(\lambda / p^{m}, \nu\right)$ are well defined elements of $\mathbb{Q} / \mathbb{Z}$. Therefore, the formulas of Theorem 5.2 are well defined. 
Proof. The decomposition

$$
\beta_{h, s}=\left(\left(\begin{array}{cc}
r & h \\
t & p^{s}
\end{array}\right), \sqrt{t \tau+p^{s}}\right) \alpha\left(\left(\begin{array}{cc}
1 & 0 \\
-p^{2 l-s} t & 1
\end{array}\right), \sqrt{-p^{2 l-s} t \tau+1}\right),
$$

where $r p^{s}-h t=1$, can easily be verified. We can choose $t$ to be positive. According to $(3.22)$

$$
\left.\mathfrak{e}_{\lambda}\right|_{L} \beta_{h, s}=\left.\left.\left.\mathfrak{e}_{\lambda}\right|_{L}\left(\left(\begin{array}{cc}
r & h \\
t & p^{s}
\end{array}\right), \sqrt{t \tau+p^{s}}\right)\right|_{L} \alpha\right|_{L} U^{-p^{2 l-s} t} .
$$

For the computation of $\left.\mathfrak{e}_{\lambda}\right|_{L}\left(\left(\begin{array}{cc}r & h \\ t & p^{s}\end{array}\right), \sqrt{t \tau+p^{s}}\right)$ we use Shintanis' formula, [Shin], Prop. 1.6.,

$$
\begin{aligned}
\left.\mathfrak{e}_{\lambda}\right|_{L}\left(\left(\begin{array}{ll}
r & h \\
t & p^{s}
\end{array}\right), \sqrt{t \tau+p^{s}}\right)=\frac{e(-\operatorname{sig}(L) / 8)^{\operatorname{sign}(-t)}}{|t|^{D / 2} \sqrt{|\mathcal{L}|}} \\
\quad \times \sum_{\mu \in \mathcal{L}} \sum_{u \in L / t L} e\left(\frac{-p^{s} b(\mu+u, \mu+u)+2 b(\mu+u, \lambda)-r b(\lambda, \lambda)}{2 t}\right) \mathfrak{e}_{\mu} .
\end{aligned}
$$

Using the equation $p^{s} r=1+h t$ the sum over $u$ can be simplified:

$$
\begin{aligned}
& \sum_{u \in L / t L} e\left(\frac{-p^{s} b(\mu+u, \mu+u)+2 b(\mu+u, \lambda)-r b(\lambda, \lambda)}{2 t}\right) \\
& =e(-h b(\mu, \lambda)+h r q(\lambda)) \sum_{u \in L / t L} e\left(\frac{-p^{s} b(\mu-r \lambda+u, \mu-r \lambda+u)}{2 t}\right)
\end{aligned}
$$

as can be checked by a straightforward calculation. It is more convenient to replace the latter sum in $(5.6)$ with a sum over the discriminant group $\mathcal{L}$. Theorem 5.5 of [Sc2] gives

$$
\sum_{r \in L / c L} e\left(-\frac{b(\alpha+r, \alpha+r)}{2 c}\right)=e(-\operatorname{sig}(L) / 8) \frac{c^{\operatorname{dim}(L) / 2}}{\sqrt{|\mathcal{L}|}} \sum_{\beta \in \mathcal{L}} e(c q(\beta)+b(\alpha, \beta)),
$$

where $\alpha \in \mathcal{L}$ and $c$ is a positive integer. Note that in order to apply formula (5.7) to the sum on the right-hand side of (5.6) one has to use the scaled quadratic form $\lambda \mapsto p^{s} q(\lambda)$. We obtain

$$
\begin{aligned}
& \sum_{u \in L / t L} e\left(\frac{-p^{s} b(\mu+u, \mu+u)+2 b(\mu+t, \lambda)-r b(\lambda, \lambda)}{2 t}\right) \\
= & \frac{t^{D / 2} e\left(-\operatorname{sig}\left(L\left(p^{s}\right)\right) / 8\right)}{\sqrt{\left|\mathcal{L}\left(p^{s}\right)\right|}} e(-h b(\mu, \lambda)+h r q(\lambda)) \sum_{\delta \in \mathcal{L}\left(p^{s}\right)} e\left(p^{s} t q(\delta)+p^{s} b(\mu-r \lambda, \delta)\right) .
\end{aligned}
$$


With the help of (3.21) and Proposition 2.1 we find for the action of $\beta_{h, s}$

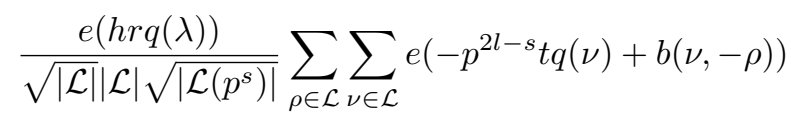

$$
\begin{aligned}
& \times \sum_{\delta \in \mathcal{L}\left(p^{s}\right)} e\left(p^{s} t q(\delta)+p^{s} b(\delta,-r \lambda)\right) \sum_{\mu \in \mathcal{L}} e\left(b\left(\mu,-h \lambda+p^{s} \delta+p^{l} \nu\right)\right) \mathfrak{e}_{\rho} \\
& =\frac{e(h r q(\lambda))}{\sqrt{|\mathcal{L}|} \sqrt{\left|\mathcal{L}\left(p^{s}\right)\right|}} \sum_{\rho \in \mathcal{L}} \sum_{\nu \in \mathcal{L}} e\left(-p^{2 l-s} t q(\nu)+b(\nu,-\rho)\right) \\
& \times \sum_{\substack{\delta \in \mathcal{L}\left(p^{s}\right) \\
p^{s} \delta=h \lambda-p^{l} \nu(\bmod L)}} e\left(p^{s} t q(\delta)+p^{s} b(\delta,-r \lambda)\right) \mathfrak{e}_{\rho}
\end{aligned}
$$

where we used for the last equation the orthogonality relations of the character $\mu \mapsto e\left(b\left(\mu, \mu^{\prime}\right)\right)$. Since $L\left(p^{s}\right)^{\prime}=\frac{1}{p^{s}} L^{\prime}$ and $\frac{1}{p^{s}} L^{\prime} / L \cong L^{\prime} / p^{s} L$ the last sum of (5.9) can be written as

$$
\sum_{\substack{\varepsilon \in L^{\prime} / p^{s} L \\ \varepsilon=h \lambda-p^{l} \nu(\bmod L)}} e\left(p^{s} t q\left(\frac{1}{p^{s}} \varepsilon\right)+b(\varepsilon,-r \lambda)\right) .
$$

Because of the isomorphism $\left(L^{\prime} / p^{s} L\right) /\left(L / p^{s} L\right) \cong \mathcal{L}$ the solution of the equation $\varepsilon=h \lambda-p^{l} \nu(\bmod L)$ is given by $h \lambda-p^{l} \nu+L / p^{s} L$. Therefore, the sum in (5.10) reads as follows

$$
\begin{aligned}
\sum_{v \in L / p^{s} L} e\left(p^{s} t q(\right. & \left.\left.\frac{1}{p^{s}}\left(v+h \lambda-p^{l} \nu\right)\right)+b\left(v+h \lambda-p^{l} \nu,-r \lambda\right)\right) \\
& =e\left(b\left(h \lambda-p^{l} \nu,-r \lambda\right)\right) \sum_{v \in L / p^{s} L} e\left(\frac{t}{p^{s}} q\left(v+h \lambda-p^{l} \nu\right)\right) .
\end{aligned}
$$

The Lemma 5.2.1 of [Ba] and Proposition 3.8 of [Sc1] combined with Theorem 5.5 of [Sc2] imply that

$$
\begin{array}{rl}
\sum_{v \in L / p^{s} L} & e\left(\frac{t}{p^{s}} q\left(v+h \lambda-p^{l} \nu\right)\right) \neq 0 \\
& \Longleftrightarrow h \lambda-p^{l} \nu \in \mathcal{L}^{p^{s}} \\
& \Longleftrightarrow \lambda \in \mathcal{L}^{p^{m}} \text { and } h \lambda / p^{m}-p^{l-m} \nu \in \mathcal{L}^{p^{s-m}}, \quad m=\min (l, s) .
\end{array}
$$

Note that $h \lambda \in \mathcal{L}^{p^{m}}$ if and only if $\lambda \in \mathcal{L}^{p^{m}}$, which follows from the identity $t h \lambda=\left(r p^{s}-1\right) \lambda$. Moreover, if $m=s$, the last condition of (5.12) simplifies to $\lambda \in \mathcal{L}^{p^{s}}$. 
If $h \lambda-p^{l} \nu \in \mathcal{L}^{p^{s}}$, the Gauss sum in (5.12) can be written as

$$
\begin{aligned}
& e\left(\frac{t}{p^{s}} q\left(h \lambda-p^{l} \nu\right)\right) \sum_{v \in L / p^{s} L} e\left(\frac{t}{p^{s}} q(v)\right) \\
& =e\left(\frac{t}{p^{s}}\left(h^{2} q(\lambda)-h p^{l} b(\lambda, \nu)+p^{2 l} q(\nu)\right)\right) \sum_{v \in L / p^{s} L} e\left(-\frac{h}{p^{s}} q(v)\right) .
\end{aligned}
$$

Replacing the sum over $\delta$ in (5.9) with the expressions of (5.11) and (5.13) respectively yields for $l \geqslant s$

$$
\begin{aligned}
\frac{\delta(\lambda, s) e\left(-\frac{h}{p^{s}} q(\lambda)\right) G_{h, s}(L)}{\sqrt{|\mathcal{L}|} \sqrt{\left|\mathcal{L}\left(p^{s}\right)\right|}} & \sum_{\rho \in \mathcal{L}} \sum_{\nu \in \mathcal{L}} e\left(b\left(\nu, p^{l-s} \lambda-\rho\right)\right) \mathfrak{e}_{\rho} \\
& =p^{-s D / 2} \delta(\lambda, s) e\left(-\frac{h}{p^{s}} q(\lambda)\right) G_{h, s}(L) \mathfrak{e}_{p^{l-s} \lambda} .
\end{aligned}
$$

Similarly, for $l<s$, because of the slightly more complicated conditions in (5.12), we obtain

$$
\begin{aligned}
& \frac{\delta(\lambda, l) G_{h, s}(L) e\left(-\frac{h}{p^{s}} q(\lambda)\right)}{\sqrt{|\mathcal{L}|} \sqrt{\left|\mathcal{L}\left(p^{s}\right)\right|}} \sum_{\rho \in \mathcal{L}} \sum_{\substack{\nu \in \mathcal{L} \\
h \lambda / p^{l}-\nu \in \mathcal{L}^{p^{s-l}}}} e\left(b\left(p^{2 l-s} \lambda / p^{l}-\rho, \nu\right)\right) \mathfrak{e}_{\rho} \\
& =\frac{\delta(\lambda, l) G_{h, s}(L) e\left(-\frac{h}{p^{s}} q(\lambda)\right)}{\sqrt{|\mathcal{L}|} \sqrt{\left|\mathcal{L}\left(p^{s}\right)\right|}} \sum_{\rho \in \mathcal{L}} e\left(b\left(p^{2 l-s} \lambda / p^{l}-\rho, h \lambda / p^{l}\right)\right) \\
& \times \sum_{\nu^{\prime} \in \mathcal{L}^{p^{s-l}}} e\left(b\left(\nu^{\prime}, p^{2 l-s} \lambda / p^{l}-\rho\right)\right) \mathfrak{e}_{\rho} .
\end{aligned}
$$

By the orthogonality relations of the character $\nu^{\prime} \mapsto e\left(b\left(\nu^{\prime}, p^{2 l-s} \lambda / p^{l}-\rho\right)\right)$ the sum over $\nu^{\prime}$ is $\left|\mathcal{L}^{p^{s-l}}\right|$, if $p^{2 l-s} \lambda / p^{l}-\rho \in \mathcal{L}_{p^{s-l}}$ and 0 otherwise. The condition $p^{2 l-s} \lambda / p^{l}-\rho \in \mathcal{L}_{p^{s-l}}$ is equivalent to $p^{s-l} \rho=\lambda$, which leads to the desired expression

$$
\frac{\delta(\lambda, l) G_{h, s}(L)\left|\mathcal{L}^{p^{s-l}}\right| e\left(\frac{h}{p^{s}} q(\lambda)\right)}{\sqrt{|\mathcal{L}|} \sqrt{\left|\mathcal{L}\left(p^{s}\right)\right|}} \sum_{\substack{\rho \in \mathcal{L} \\ p^{s-l} \rho=\lambda}} e\left(b\left(-\rho, h \lambda / p^{l}\right)\right) \mathfrak{e}_{\rho} .
$$

Theorem 5.4. Let $p$ be an odd prime, $l$ a positive integer and $f \in M_{k, L}$ with $k \in \frac{1}{2} \mathbb{Z}$ and the Fourier expansion

$$
f(\tau)=\sum_{\lambda \in \mathcal{L}} \sum_{\substack{n \in \mathbb{Z}+q(\lambda) \\ n \geqslant 0}} c(\lambda, n) e(n \tau) \mathfrak{e}_{\lambda} .
$$

Further, let $G_{s}(L)$ be the Gauss sum $\sum_{v \in L / p^{s} L} e\left(\frac{1}{p^{s}} q(v)\right), D=\operatorname{dim}(L)$ and write

$$
K_{1}(L, s)=p^{(2 k-2) l} p^{-s D / 2} p^{-s k} G_{s}(L)
$$


and

$$
K_{2}(L, s)=p^{(2 k-2) l} p^{-s D / 2} p^{-s k} G_{s}(L)\left|\mathcal{L}_{s-l}\right|^{-1} .
$$

Then

$$
\left.f\right|_{k, L} T\left(p^{2 l}\right)=\sum_{\lambda \in \mathcal{L}} \sum_{\substack{n \in \mathbb{Z}+q(\lambda) \\ n \geqslant 0}} b(\lambda, n) e(n \tau) \mathfrak{e}_{\lambda},
$$

where

$$
\begin{aligned}
b(\lambda, n)= & \delta(\lambda, l) \sum_{\substack{\lambda^{\prime} \in \mathcal{L}_{p^{l}} \\
n-p^{2 l} q\left(\lambda / p^{l}+\lambda^{\prime}\right) \in p^{2 l} \mathbb{Z}}} c\left(\lambda / p^{l}+\lambda^{\prime}, \frac{n-p^{2 l} q\left(\lambda / p^{l}+\lambda^{\prime}\right)}{p^{2 l}}+q\left(\lambda / p^{l}+\lambda^{\prime}\right)\right) \\
& +\sum_{s=1}^{l} \delta(\lambda, l) K_{1}(L, s) \\
& \times \sum_{\substack{\lambda^{\prime} \in\left(\mathcal{L}^{p^{s}}\right)_{p^{l-s}}\\
}} g\left(p^{s}, \chi_{p}^{R}, \frac{n-p^{2(l-s)} q\left(\lambda / p^{l-s}+\lambda^{\prime}\right)}{\left.p^{2(l-s)}\right)}\right) \\
& \times c\left(\lambda / p^{l(l-s)} q\left(\lambda / p^{l-s}+\lambda^{\prime}\right) \in p^{2(l-s)} \mathbb{Z}\right. \\
& +\sum_{s=l+1}^{2 l-1} \delta\left(p^{s-l} \lambda, \frac{n-p^{2(l-s)} q\left(\lambda / p^{l-s}+\lambda^{\prime}\right)}{\left.p^{2(l-s)}+q\left(\lambda / p^{l-s}+\lambda^{\prime}\right)\right)}\right. \\
& +c\left(p^{l} \lambda, p^{2 l} n\right) .
\end{aligned}
$$

Here $R=\sum_{i=0}^{s-1}(s-i) n_{i}$ with $n_{i}$ defined in Lemma 4.5, $\delta(\lambda, x)$ is defined in Theorem 5.2 and $\chi_{p}=(\dot{\dot{p}})$.

Remark 5.5. It can be extracted directly from the following proof that the group $\left(\mathcal{L}^{p^{s}}\right)_{p^{l-s}}$ for $s=l$ in $(5.15)$ has to be interpreted as the zero element of $\mathcal{L}$. In order to have (5.15) as simple as possible we do not specify a separate formula for the case $s=l$.

Proof. Using (5.1) the definition (3.23) yields

$$
\begin{aligned}
\left.f\right|_{k, L} T\left(p^{2 l}\right)= & p^{l(k-2)}\left(\sum_{\lambda \in \mathcal{L}}\left(\left.f_{\lambda}\right|_{k} \alpha\right)\left(\left.\mathfrak{e}_{\lambda}\right|_{L} \alpha\right)\right. \\
& +\sum_{s=1}^{2 l-1} \sum_{h \in\left(\mathbb{Z} / p^{s} \mathbb{Z}\right)^{*}} \sum_{\lambda \in \mathcal{L}}\left(\left.f_{\lambda}\right|_{k} \beta_{h, s}\right)\left(\left.\mathfrak{e}_{\lambda}\right|_{L} \beta_{h, s}\right) \\
& \left.+\sum_{b \in Z / p^{2 l} \mathbb{Z} \lambda \in \mathcal{L}} \sum_{\lambda}\left(\left.f_{\lambda}\right|_{k} \gamma_{b}\right)\left(\left.\mathfrak{e}_{\lambda}\right|_{L} \gamma_{b}\right)\right)
\end{aligned}
$$


For (5.16b) the formulas of Theorem 5.2 suggest to distinguish between the two cases $s \leqslant l$ and $s>l$.

Lemma 4.5 can be applied to the sum $G_{h, s}(L)$ which occurs in the formulas of Theorem 5.2. We obtain

$$
G_{h, s}(L)=\chi_{p}^{R}(h) G_{s}(L) .
$$

This identity will be used in the following for both of the above mentioned cases.

First, we consider the case $s \leqslant l$.

$$
\begin{aligned}
p^{l(k-2)} & \sum_{h \in\left(\mathbb{Z} / p^{s} \mathbb{Z}\right)^{*}} \sum_{\lambda \in \mathcal{L}}\left(\left.f_{\lambda}\right|_{k} \beta_{h, s}\right)\left(\left.\mathfrak{e}_{\lambda}\right|_{L} \beta_{h, s}\right) \\
= & p^{-s D / 2} p^{(k-2) l} p^{-s k} \\
& \times \sum_{h \in\left(\mathbb{Z} / p^{s} \mathbb{Z}\right)^{*}} \sum_{\lambda \in \mathcal{L}} f_{\lambda}\left(\frac{p^{2 l-s} \tau+h}{p^{s}}\right) \delta(\lambda, s) e\left(-\frac{h}{p^{s}} q(\lambda)\right) G_{h, s}(L) \mathfrak{e}_{p^{l-s} \lambda} \\
= & K_{1}(L, s) \sum_{\lambda \in \mathcal{L}^{p}}\left(\sum_{n \in \mathbb{Z}+q(\lambda)} c(\lambda, n) g\left(p^{s}, \chi_{p}^{R}, n-q(\lambda)\right) e\left(\frac{p^{2 l-s} n \tau}{p^{s}}\right)\right) \mathfrak{e}_{p^{l-s} \lambda},
\end{aligned}
$$

where $g\left(p^{s}, \chi_{p}^{R}, n-q(\lambda)\right)$ is one of the quadratic Gauss sums defined in Lemma 4.1. By the exact sequence $(2.1)$ applied to $\mathcal{L}^{p^{s}}$ every $\lambda \in \mathcal{L}^{p^{s}}$ can be written as $\nu / p^{l-s}+\nu^{\prime}$ where $\nu \in \mathcal{L}^{p^{l}}$ and $\nu^{\prime} \in\left(\mathcal{L}^{p^{s}}\right)_{p^{l-s}}$. We therefore obtain for the last expression above

$$
\begin{aligned}
& K_{1}(L, s) \sum_{\nu \in \mathcal{L}^{p^{l}}} \sum_{\nu^{\prime} \in\left(\mathcal{L}^{p^{s}}\right)_{p^{l-s}}} \sum_{n \in \mathbb{Z}+q\left(\nu / p^{l-s}+\nu^{\prime}\right)} c\left(\nu / p^{l-s}+\nu^{\prime}, n\right) \\
& \left.\times g\left(p^{s}, \chi_{p}^{R}, n-q\left(\nu / p^{l-s}+\nu^{\prime}\right)\right)\right) e\left(p^{2(l-s)} n \tau\right) \mathfrak{e}_{\nu} \sum K_{1}(L, s) \sum_{\nu \in \mathcal{L}^{p^{l}}} \sum_{\nu^{\prime} \in\left(\mathcal{L}^{p^{s}}\right)_{p^{l-s}}} c\left(\nu / p^{l-s}+\nu^{\prime}, n / p^{2(l-s)}\left(\mathbb{Z}+q\left(\nu / p^{l-s}+\nu^{\prime}\right)\right)\right. \\
& \times g\left(p^{s}, \chi_{p}^{R}, n / p^{2(l-s)}-q\left(\nu / p^{l-s}+\nu^{\prime}\right)\right) e(n \tau) \mathfrak{e}_{\nu} .
\end{aligned}
$$

Any $n=p^{2(l-s)}\left(r+q\left(\nu / p^{l-s}+\nu^{\prime}\right)\right) \in p^{2(l-s)}\left(\mathbb{Z}+q\left(\nu / p^{l-s}+\nu^{\prime}\right)\right)$ can be understood as an element of $\mathbb{Z}+q(\nu)$ where the integer part is given by $p^{2(l-s)} r+p^{l-s} b\left(\nu, \nu^{\prime}\right)+$ $p^{2(l-s)} q\left(\nu^{\prime}\right)$. Summing over $\mathbb{Z}+q(\nu)$, the last sum of (5.18) can be written as

$$
\begin{aligned}
K_{1}(L, s) \sum_{\nu \in \mathcal{L}^{p^{l}}} & \sum_{n \in \mathbb{Z}+q(\nu)} \sum_{\substack{\nu^{\prime} \in\left(\mathcal{L}^{p^{s}}\right)_{p^{l-s}} \\
q-p^{2(l-s)} q\left(\nu / p^{l-s}+\nu^{\prime}\right) \in p^{2(l-s)} \mathbb{Z}}} \\
& \times c\left(\nu / p^{l-s}+\nu^{\prime}, \frac{n-p^{2(l-s)} q\left(\nu / p^{l-s}+\nu^{\prime}\right)}{p^{2(l-s)}}+q\left(\nu / p^{l-s}+\nu^{\prime}\right)\right) \\
& \times g\left(p^{s}, \chi_{p}^{R}, \frac{n-p^{2(l-s)} q\left(\nu / p^{l-s}+\nu^{\prime}\right)}{p^{2(l-s)}}\right) e(n \tau) \mathfrak{e}_{\nu} .
\end{aligned}
$$


It remains to treat the case $l<s$. Theorem 5.2 combined with identity (5.17) yields

$$
\begin{aligned}
p^{l(k-2)} & \sum_{h \in\left(\mathbb{Z} / p^{s} \mathbb{Z}\right)^{*}} \sum_{\lambda \in \mathcal{L}}\left(\left.f_{\lambda}\right|_{k} \beta_{h, s}\right)\left(\left.\mathfrak{e}_{\lambda}\right|_{L} \beta_{h, s}\right) \\
= & K_{2}(L, s) \sum_{h \in\left(\mathbb{Z} / p^{s} \mathbb{Z}\right)^{*}} \sum_{\lambda \in \mathcal{L}} f_{\lambda}\left(\frac{p^{2 l-s} \tau+h}{p^{s}}\right) \delta(\lambda, l) \chi_{p}(h)^{R} e\left(-\frac{h}{p^{s}} q(\lambda)\right) \\
& \times \sum_{\substack{\varrho \in \mathcal{L} \\
p^{s-l} \varrho=\lambda}} e\left(b\left(-\varrho, h \lambda / p^{l}\right) \mathfrak{e}_{\varrho} .\right.
\end{aligned}
$$

By a straightforward calculation, using the fact $p^{s-l} \rho=\lambda$, we find $e\left(\frac{h}{p^{s}} q(\lambda)\right) e\left(-b\left(\rho, h \lambda / p^{l}\right)\right)=e\left(-\frac{h}{p^{s}} q(\lambda)\right)$. The last expression above can then be written as

$$
\begin{aligned}
& K_{2}(L, s) \sum_{\lambda \in \mathcal{L}^{p^{l}}} \sum_{\substack{\rho \in \mathcal{L} \\
p^{s-l} \rho=\lambda}} \\
& \times \sum_{n \in \mathbb{Z}+q(\lambda)} c(\lambda, n)\left(\sum_{h \in\left(\mathbb{Z} / p^{s} \mathbb{Z}\right)^{*}} \chi_{p}(h)^{R} e\left(\frac{h(n-q(\lambda))}{p^{s}}\right)\right) e\left(n \tau / p^{2(s-l)}\right) \mathfrak{e}_{\rho} .
\end{aligned}
$$

The equation $p^{s-l} \rho=\lambda$ has a solution if $\lambda \in \mathcal{L}^{p^{s-l}}$. This is in particular fulfilled for all $\lambda \in \mathcal{L}^{p^{l}}$ since $l>s-l$. The set of solutions is given by $\lambda / p^{s-l}+\mathcal{L}_{p^{s-l}}$. We obtain

$$
K_{2}(L, s) \sum_{\lambda \in \mathcal{L}^{p^{l}}} \sum_{\lambda \mathcal{\lambda}^{\prime} \in \mathcal{L}_{p^{s-l}}} \sum_{n \in \mathbb{Z}+q(\lambda)} c(\lambda, n) g\left(p^{s}, \chi_{p}^{R}, n-q(\lambda)\right) e\left(n \tau / p^{2(s-l)}\right) \mathfrak{e}_{\lambda / p^{s-l}+\lambda^{\prime}}
$$

By the exact sequence (2.1) we can write each element of $\mathcal{L}$ as $\lambda / p^{s-l}+\lambda^{\prime}$ and therefore replace the sums over $\mathcal{L}^{p^{l}}$ and $\mathcal{L}_{p^{s-l}}$ with a sum over $\mathcal{L}$ :

$$
K_{2}(L, s) \sum_{\substack{\mu \in \mathcal{L} \\ p^{s-l} \mu \in \mathcal{L}^{p^{l}}}} \sum_{n \in \mathbb{Z}+q\left(p^{s-l} \mu\right)} c\left(p^{s-l} \mu, n\right) g\left(p^{s}, \chi_{p}^{R}, n-q\left(p^{s-l} \mu\right)\right) e\left(n \tau / p^{2(s-l)}\right) \mathfrak{e}_{\mu} .
$$

From Lemma 4.1 it can be deduced that the quadratic Gauss sum $g\left(p^{s}, \chi_{p}^{R}, n-\right.$ $\left.q\left(p^{s-l} \mu\right)\right)$ is zero unless $p^{s-1} \mid\left(n-q\left(p^{s-l} \mu\right)\right)$. Since $s-1 \geqslant 2(s-l)$ this is in particular satisfied for all $n$ with $n-q\left(p^{s-l} \mu\right) \in p^{2(s-l)} \mathbb{Z}$. Taking this into account we can replace $n$ with $p^{2(s-l)} m$ where $m$ runs through $\mathbb{Z}+q(\mu)$. We obtain

$$
K_{2}(L, s) \sum_{\substack{\mu \in \mathcal{L} \\ p^{s-l} \mu \in \mathcal{L}^{p^{l}}}} \sum_{m \in \mathbb{Z}+q(\mu)} c\left(p^{s-l} \mu, p^{2(s-l)} m\right) g\left(p^{s}, \chi_{p}^{R}, p^{2(s-l)}(m-q(\mu))\right) e(m \tau) \mathfrak{e}_{\mu} .
$$

The contributions to the Fourier expansion coming from $\alpha$ and $\gamma_{b}$ can be treated exactly in the same way as the contributions coming from the corresponding matrices in proof of Theorem 14.2.9 in [St]. 
The following corollary provides the Fourier expansion of the Hecke operator $T\left(p^{2 l}\right)$ in the important special case where $p$ is coprime to the level of $L$. It generalises Proposition 4.3 and Theorem 4.10 of [BS].

Corollary 5.6. Let $p$ be an odd prime which is coprime to the level $N$ of the lattice $L$ and $l \in \mathbb{N}$. Let $f \in M_{k, L}$ with the Fourier expansion

$$
f(\tau)=\sum_{\lambda \in \mathcal{L}} \sum_{\substack{n \in \mathbb{Z}+q(\lambda) \\ n \geqslant 0}} c(\lambda, n) e(n \tau) \mathfrak{e}_{\lambda} .
$$

Then

$$
\left.f\right|_{k, L} T\left(p^{2 l}\right)=\sum_{\lambda \in \mathcal{L}} \sum_{\substack{n \in \mathbb{Z}+q(\lambda) \\ n \geqslant 0}} b(\lambda, n) e(n \tau) \mathfrak{e}_{\lambda}
$$

with

$$
\begin{aligned}
b(\lambda, n)= & p^{(2 k-2) l} c\left(\lambda / p^{l}, \frac{n-p^{2 l} q\left(\lambda / p^{l}\right)}{p^{2 l}}+q\left(\lambda / p^{l}\right)\right) \\
& +p^{(2 k-2) l} \sum_{s=1}^{l} p^{-s k} \varepsilon_{p^{s}}^{D}\left(\frac{(-1)^{b^{-}}|\mathcal{L}|}{p^{s}}\right)\left(\frac{2}{p^{s}}\right)^{D} \\
& \times c\left(\lambda / p^{l-s}, \frac{n-p^{2(l-s)} q\left(\lambda / p^{l-s}\right)}{p^{2(l-s)}}+q\left(\lambda / p^{l-s}\right)\right) \\
& \times g\left(p^{s}, \chi_{p}^{D}, \frac{n-p^{2(l-s)} q\left(\lambda / p^{l-s}\right)}{p^{2(l-s)}}\right) \\
& +p^{(2 k-2) l} \sum_{s=l+1}^{2 l-1} \varepsilon_{p^{s}}^{D}\left(\frac{(-1)^{b^{-}}|\mathcal{L}|}{p^{s}}\right)\left(\frac{2}{p^{s}}\right)^{D} \\
& \times c\left(p^{s-l} \lambda, p^{2(s-l)} n\right) g\left(p^{s}, \chi_{p}^{D}, n-q\left(p^{s-l} \lambda\right)\right) \\
& +c\left(p^{l} \lambda, p^{2 l} n\right) .
\end{aligned}
$$

Here, $c\left(\lambda / p^{l}, \frac{n-p^{2 l} q\left(\lambda / p^{l}\right)}{p^{2 l}}+q\left(\lambda / p^{l}\right)\right)$ is zero unless $n-p^{2 l} q\left(\lambda / p^{l}\right) \in p^{2 l}$ and accordingly $c\left(\lambda / p^{l-s}, \frac{n-p^{2(l-s)} q\left(\lambda / p^{l-s}\right)}{p^{2(l-s)}}+q\left(\lambda / p^{l-s}\right)\right)$ is zero unless $n-p^{2(l-s)} q\left(\lambda / p^{l-s}\right) \in$ $p^{2(l-s)} \mathbb{Z}$.

Proof. Since by the assumption $(p, N)=1$ and each prime divisor of $N$ is also one of $|\mathcal{L}|, p$ and $|\mathcal{L}|$ are also coprime. Therefore,

$$
\mathcal{L}^{p^{s}}=\mathcal{L}
$$

and

$$
\mathcal{L}_{p^{s}}=\{0\}
$$

for all $s=1, \ldots, 2 l-1$. Taking this and the explicit formula (4.11) into account, the Fourier expansion (5.19) follows immediately from the general expansion in (5.15). 
The following corollary shows that the definition of Hecke operators given in [BS] and the calculated Fourier expansion in Theorem 5.4 is compatible with Hecke operators for Jacobi forms. Note that we only consider the special case $l=1$ of Theorem 5.4 since the comparison in the general case is very tedious.

Corollary 5.7. Let $k$ be an integer, $m \in \mathbb{N}$. Further, set $L=\mathbb{Z}$ with the quadratic form $q(x)=-m x^{2}$. The associated bilinear form is $b(x, y)=-2 m x y$. With this choice of $(L, b)$ one can easily show that $\mathcal{L}=\frac{1}{2 m} \mathbb{Z} / \mathbb{Z} \cong \mathbb{Z} / 2 m \mathbb{Z}$ and $M_{k-1 / 2, L}$ is isomorphic to the space $J_{k, m}$ of Jacobi forms of weight $k$ and index $m$. Denote with $I$ the beforehand mentioned isomorphism. Then for all odd primes $p$ and all $f \in M_{k-1 / 2, L}$ the following identity holds

$$
I\left(\left.f\right|_{k-1 / 2} T\left(p^{2}\right)\right)=\left(I(f) \mid T_{p}\right)
$$

where $T_{p}$ denotes the Hecke operator on $J_{k, m}$.

Proof. A detailed proof can be found in [St], Bemerkung 14.2.12.

\section{References}

[Ba] A. Barnard, The Singular Theta Correspondence, Lorentzian Lattices and Borcherds-Kac-Moody Algebras, Ph.D. Dissertation, U.C. Berkeley, 2003.

[BE] B. Berndt, R. Evans and K. Williams, Gauss and Jacobi sums, Canadian Mathematical Society series of monographs and advanced texts, 21, Wiley, 1998.

[Bo1] R. Borcherds, Automorphic forms with singularities on Grassmannians, Inv. Math. 132 (1998), 491-562.

[Bo2] R. Borcherds, Reflection groups of Lorentian lattices, Duke Math. J. 104 (2000), 319-366.

[Br] J.H. Bruinier, Borcherds products on $\mathrm{O}(2, l)$ and Chern classes of Heegner divisors, Springer Lecture Notes in Mathematics 1780, Springer-Verlag, 2002.

[BO1] J.H. Bruinier and K. Ono, Heegner Divisors, L-functions and harmonic weak Maass forms, Annals of Math. 172 (2010), 2135-2181.

[BO2] J.H. Bruinier and K. Ono, Algebraic formulas for the coefficients of half integral weight harmonic weak Mass forms, preprint (2011).

[BS] J.H. Bruinier and O. Stein, The Weil representation and Hecke operators on vector-valued modular forms, Math. Z. 264 (2010), 249-270.

[Bu] M. Bundschuh, Über die Endlichkeit der Klassenzahl gerader Gitter der Signatur $(2, n)$ mit einfachem Kontrollraum, Dissertation, Universität Heidelberg, 2001.

[CS] J.H. Conway and H.J. Sloane, Sphere packings, lattices and groups, third edition. With additional contributions by E. Bannai, R.E. Borcherds, J. Leech, S.P. Norton, A.M. Odlyzko, R.A. Parker, L. Queen and B.B. Venkov. Grundlehren der Mathematischen Wissenschaften 290, Springer-Verlag, New York, 1999. 
[Eb] W. Ebeling, Lattices and codes, a course partially based on lectures by F. Hirzebruch, second revised edition, Advanced Lectures in Mathematics, Vieweg, Braunschweig, 2002.

[EZ] M. Eichler and D. Zagier, The Theory of Jacobi Forms, Progress in Math. 55, Birkhäuser, 1985.

[KS] M. Kneser, Quadratische Formen, Neu bearbeitet und herausgegeben in Zusammenarbeitet mit Rudolf Scharlau, Springer Verlag, 2001.

[NW] A. Nobs and J. Wolfart, Die irreduziblen Darstellungen der Gruppen $\mathrm{SL}_{2}\left(\mathbb{Z}_{p}\right)$, insbesondere $\mathrm{SL}_{2}\left(\mathbb{Z}_{2}\right)$, I. Teil, Comment Math. Helvetici 51 (1976), 491-526.

[Sc] N.R. Scheithauer, On the classification of automorphic products and generalized Kac-Moody algebras, Invent. Math. 164 (2006), 641-678.

[Sc1] N.R. Scheithauer, The Weil representation of $\mathrm{SL}_{2}(\mathbb{Z})$ and some applications, Int. Math. Res. Not. 8 (2009), 1488-1545.

[Sc2] N.R. Scheithauer, Moonshine for Conway's group, Habilitationsschrift, Ruprecht-Karls-Universität Heidelberg, 2004.

[Sh1] G. Shimura, Introduction to the Arithmetic Theory of Automorphic Functions, Princeton University Press, Princeton, 1971.

[Sh2] G. Shimura, On modular forms of half integral weight, Annals of Math. 97 (1973), 440-481.

[Sh3] G. Shimura, The special values of the zeta functions associated to Hilbert modular forms, Duke Math. J. 45 (1978), no. 3, 637-679.

[Shin] T. Shintani, On the construction of holomorphic cusp forms of half integral weight, Nagoya Math. J. 58 (1975), 83-126.

[St] O. Stein, Hecke-Operatoren und vektorwertige Modulformen zur Weildarstellung, Dissertation, Universität zu Köln, 2007.

[Str] F. Strömberg, Weil representations associated to finite quadratic modules, Math. Z. 275 (2013), no. 2, 509-527.

Address: Oliver Stein: Ostbayerische Technische Hochschule Regensburg, Fakultät Informatik und Mathematik, Universitätsstraße 31, 93053 Regensburg, Germany.

E-mail: oliver.stein@hs-regensburg.de

Received: 27 March 2014; revised: 6 April 2014 